\title{
A Fundamental Role of the Molecular Length in Forming Metal-Organic Hybrids of Phenol Derivatives on Silver Surfaces
}

Yangyang Han', Junbo Wang ${ }^{\dagger}$, Luying Song ${ }^{\dagger}$, Yuanjing Zheng ${ }^{\dagger}$, Youyong Lił, Haiping $\operatorname{Lin}^{*},+,+$, Qing $L i^{*},+,+$ Lifeng $C h i^{*}+*$

$\dagger$ Institute of Functional Nano \& Soft Materials (FUNSOM), Jiangsu Key Laboratory for Carbon-Based Functional Materials \& Devices, Soochow University, 199 Ren'ai Road, Suzhou, 215123 P. R. China

${ }^{\star}$ School of Physics and Information Technology, Shaanxi Normal University, Xi'an 710119, China

\section{Methods}

STM Measurements. All the experiments were performed with commercial low temperature scanning tunneling microscopy (Unisoku LT-STM 1500s) at a base pressure better than $1 \times 10^{-10}$ Torr. The $\mathrm{Ag}(100)$ surface was prepared by several cycles of sputtering with argon ions and subsequent annealing at $730 \mathrm{~K}$. A tungsten tip was used for the STM imaging. All images were processed and analyzed by WSxM. DHTP molecules (purity higher than 99\%) were purchased from HWRK CHEM Company. 
DHBP molecules (purity higher than 99\%) were purchased from Adamas Company.

Theoretical Calculations. The density functional theory (DFT) calculations were carried out using the Vienna Ab-initio Simulation Package (VASP version 5.4). ${ }^{1-}$

${ }^{2}$ The exchange-correlation functional was described by the Perdew, Burke, and Ernzerhof (PBE) parameterization of the generalised gradient approximation (GGA). ${ }^{3}$ The interactions between the electrons and the ions were treated within the projector augmented-wave (PAW) approximation. ${ }^{4-5}$ A conjugate-gradient algorithm was used to relax the atoms into their instantaneous ground state positions. The $\operatorname{Ag}(100)$ surface was mimicked by a $(5 \times 10)$ slab model consisting of five atomic layers. The bottom three layers of Ag atoms were kept fixed. The structural optimizations were not stopped until the atomic forces were less than $0.01 \mathrm{eV} / \AA$. The first irreducible Brillouin zone was modelled based on the Gamma-centered Monkhorst-Pack scheme, where a $4 \times 2 \times 1$ k-points grid was used in geometry optimizations and electronic structure analysis. The vdW-D3 method was adopted to describe the van der Waals interactions. ${ }^{6}$ A vacuum of $20 \AA$ was employed to avoid the interactions between periodic images in $\mathrm{z}$ direction.

\section{1, Optimized structures on a Ag(100) surface.}

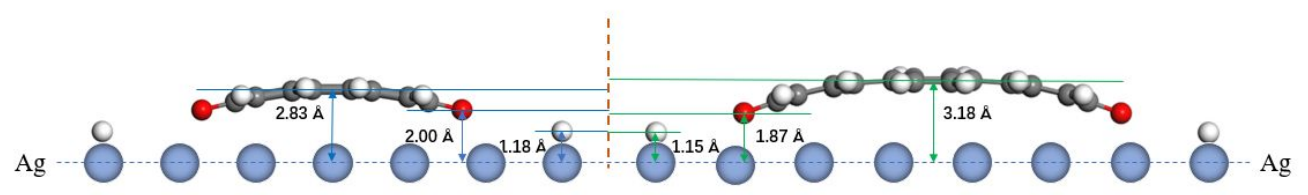

Figure S1. The optimized structures of deprotonated DHBP and DHTP on a $\operatorname{Ag}(100)$ 
surface.

\section{2, Molecular strain of dehydrogenated DHBP and DHTP on $\operatorname{Ag}(100)$}

The molecular strains resulted from the binding of dehydrogenated DHBP and DHTP on $\operatorname{Ag}(100)$ are estimated by $E_{\text {strain }}=E_{\text {free }}-E_{\text {adsorb }}$. Here, $E_{\text {free }}$ is the energy of a freestanding molecule in the vacuum, while $E_{\text {adsorb }}$ is the energy of a bended molecule (the same configuration as it is on the Ag surface) in the vacuum.

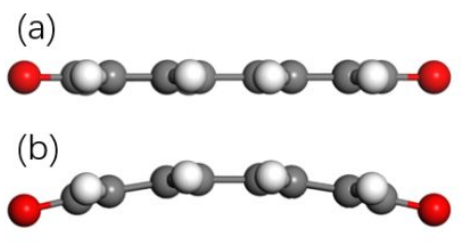

$\Delta \mathrm{E}=0.68 \mathrm{eV}$

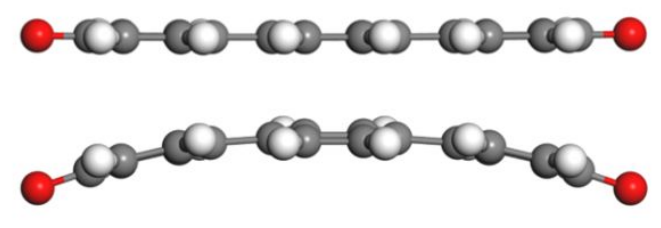

$\Delta \mathrm{E}=0.54 \mathrm{eV}$

Figure S2. The configurations of dehydrogenated DHBP and DHTP under vacuum (a) without adsorption strain; (b) with adsorption strain on $\mathrm{Ag}$ (100). The value is the calculated $E_{\text {strain }}$.

\section{3, The binding configuration of pristine DHBP and DHTP on $\operatorname{Ag(100)}$}

As shown in Figure S3, the molecular backbone of pristine DHBP and DHTP are straight and in a flat fashion when they are adsorbed on $\operatorname{Ag}(100)$. 
(a)

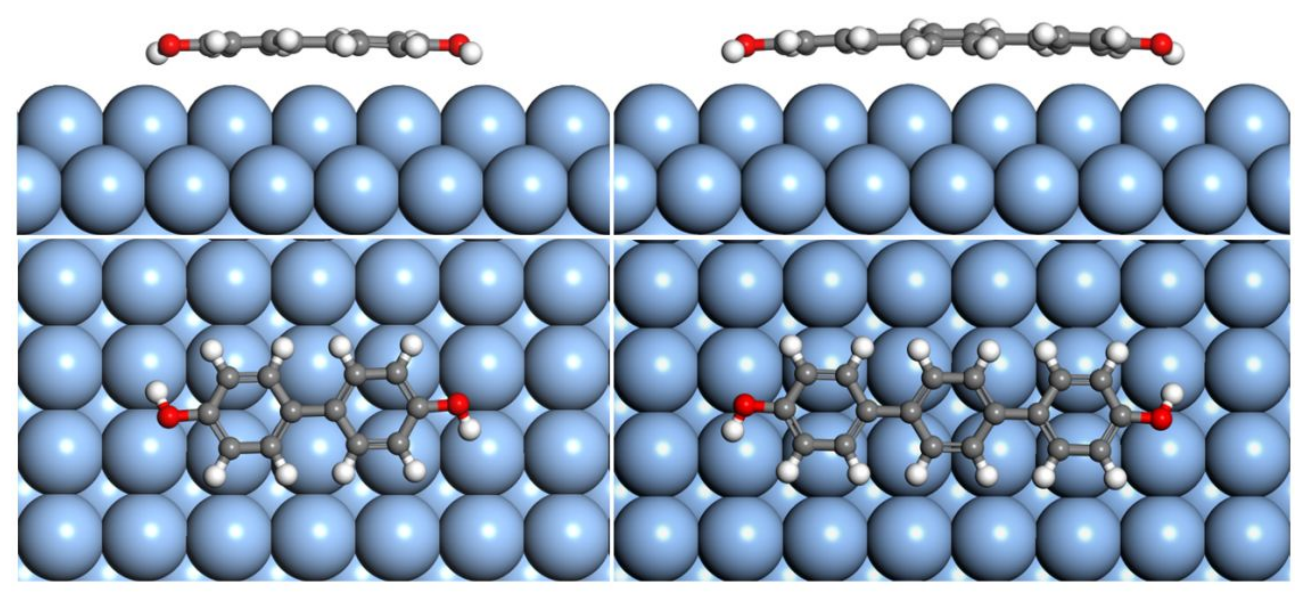

Figure S3. The configurations of pristine (a) DHBP and (b) DHTP adsorbing on Ag (100) surfaces.

\section{4, Self-assembly of DON on $\operatorname{Ag(100)}$}

After depositing 2,6-Dihydroxynaphthalene (DON) molecule on a $\operatorname{Ag}(100)$ surface held at $540 \mathrm{~K}$, metal-organic hybrids are observed on the surface, as shown in Figure S4a. Closer investigation reveals that each silver adatom bonds with four oxygen atoms, forming tetramer intermediates (Figure S4b).
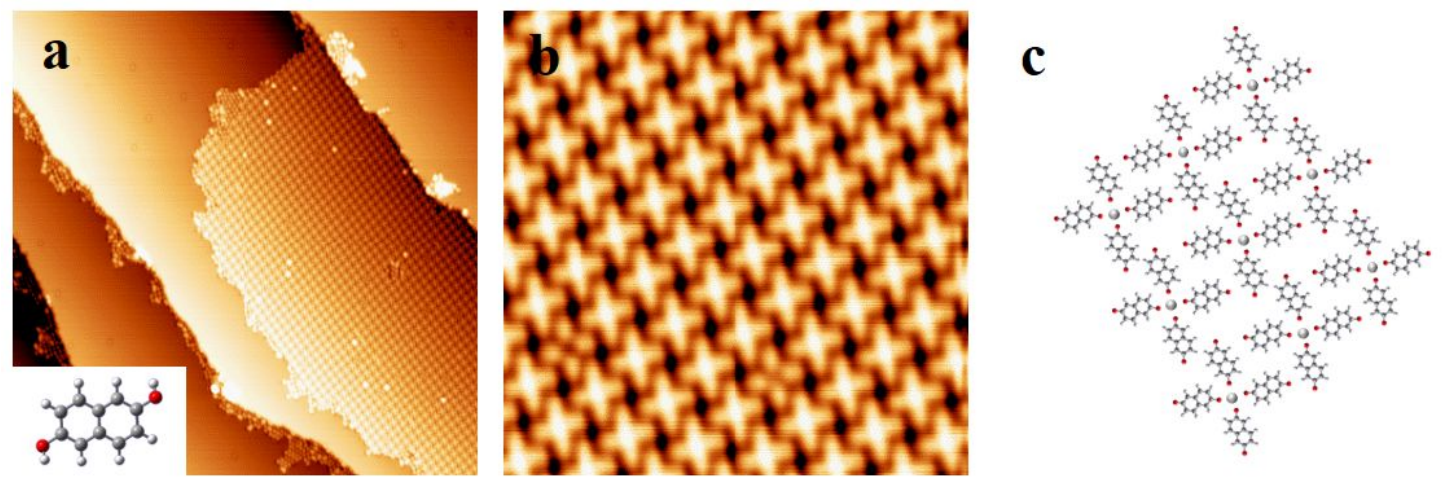

Figure S4. (a) large scale STM image after depositing DON molecule onto a $\mathrm{Ag}(100)$ surface held at $540 \mathrm{~K}$. The inset is the structural model of DON (b) the zoomed image 
of (a). The image size is $100 \times 100 \mathrm{~nm}$ for (a) and $15 \times 15 \mathrm{~nm}$ for (b). Tunneling parameters are $I_{\mathrm{t}}=20 \mathrm{pA}, V_{\mathrm{b}}=-1 \mathrm{~V}$ for $(\mathrm{a}) ; I_{\mathrm{t}}=100 \mathrm{pA}, V_{\mathrm{b}}=-1 \mathrm{~V}$ for $(\mathrm{b})$.

\section{References}

(1) Kohn, W.; Becke, A. D.; Parr, R. G. Density Functional Theory of Electronic Structure. J. Phys. Chem. 1996, 100, 12974-12980.

(2) Kresse, G.; Furthmuller, J. Efficient Iterative Schemes for ab Initio Total-energy Calculations Using a Plane-wave Basis Set. Phys. Rev. B 1996, 54, 11169-11186.

(3) Perdew, J. P.; Burke, K.; Ernzerhof, M. Generalized Gradient Approximation Made Simple. Phys. Rev. Lett. 1996, 77, 3865-3868.

(4) Blochl, P. E. Projector Augmented-wave Method. Phys. Rev. B 1994, 50, 17953 17979.

(5) Kresse, G.; Joubert, D. From Ultrasoft Pseudopotentials to the Projector Augmented-wave Method. Phys. Rev. B 1999, 59, 1758-1775.

(6) Grimme, S.; Ehrlich, S.; Goerigk, L. Effect of the Damping Function in Dispersion Corrected Density Functional Theory. J. Comput. Chem. 2011, 32, 1456-1465. 